\title{
Three Sesquiterpenoid Dimers from Chloranthus japonicus. Absolute Configuration of Chlorahololide A and Related Compounds
}

Abstract: A novel sesquiterpenoid dimer, named multistalide C (1), together with two known congeners, shizukaols C (2) and $D(3)$, was isolated from the whole plant of Chloranthus japonicus Sieb. The structures of compounds 1-3 were elucidated by extensive HR-ESI-MS, 1D and 2D NMR spectroscopic analysis. Compounds 1-3 exhibited significant toxic effects on brine shrimp larvae (Artemia salina).
The absolute configuration of $\mathbf{1}$ was established by CD/TDDFT calculations. The related compound chlorahololide A was also reinvestigated. The previous assignment of the absolute configuration of chlorahololide $A$ and several related sesquiterpenoid dimers, based on an incorrect application of the exciton chirality method, is criticized.

\section{Xin-Wei Shi, ${ }^{*[a]}$ Qiang-Qiang Lu, ${ }^{[a]}$ Gennaro Pescitelli, ${ }^{\left[{ }^{[b]}\right]}$ Trpimir Ivšić, ${ }^{[b, c]}$ Jun-Hui Zhou, ${ }^{[a]}$ and Jin-Ming Gao*[d]}

Keywords: Natural products; Structure elucidation; Electronic Circular Dichroism; TDDFT calculations; Exciton Chirality Method; Brine shrimp larvae toxicity

\section{Introduction}

The genus Chloranthus (Chloranthaceae) is mainly distributed over the east of Asia, and it comprises 13 species and five varieties in China. ${ }^{1,2}$ Chloranthus japonicus Sieb ('yin-xian-cao' in Chinese) has been long used in traditional Chinese medicine for the treatment of bone fractures, trauma, rheumatism, and cough. $^{3-5}$ The previous phytochemical investigations on this plant have resulted in the isolation of a number of sesquiterpenoid dimers. ${ }^{6-9}$ Some of them were reported to show multi-biological activities, such as antifungal, potent and selective tumor growth-inhibitory, inhibition of cell adhesion molecule expression and tyrosinase. ${ }^{10-12}$

As part of our ongoing search for new bioactive natural products from the traditional Chinese herbal medicines in the Qinba Mountains, ${ }^{13-17}$ chemical studies of the whole plant of $C$. japonicus were carried out, leading to the discovery of a new sesquiterpenoid dimer, named multistalide $C(\mathbf{1})$, together with two known compounds, shizukaols $C$ and D (2 and 3 ) (Chart 1$)$. Herein, we describe the isolation, structural identification, including the absolute configuration, and brine shrimp larvae toxicity of the three compounds. Since compound $\mathbf{1}$ is structurally related to chlorahololide A (4), this latter compound was also reconsidered. In fact, the absolute configuration of chlorahololide $\mathrm{A}^{18}$ and several related sesquiterpenoid dimers has been assigned using the exciton chirality method, ${ }^{19-21}$ but without the necessary prerequisites for the application of this well-known approach. The limitations of the exciton chirality method for this family of chlorahololide analogues are discussed in details.

\section{Materials and Methods}

General experimental procedures: NMR Spectra were recorded with a Bruker AV-500 instrument, using TMS as internal standard. Mass spectra (HR-ESI-MS and ESI-MS) were recorded with a ThermoFisher Q-Exactive spectrometer. IR spectra were recorded with a Shimadzu FTIR-8900 instrument. UV spectra were recorded with a Shimadzu UV-2401A spectrophotometer. ECD spectra were recorded with a Jasco J-
810 spectropolarimeter. Optical rotations were measured with a Rudolph Autopol-Automatic polarimeter, Silica gel $\left(\mathrm{SiO}_{2}: 100-\right.$ 200, 200-300 mesh) was acquired from Qingdao Marine, China. $\mathrm{MCl}$ gel $(75-150 \mu \mathrm{m})$ was acquired from Mitsubishi, Japan. HPLC separations were run with RP-18 (LiChroprep, 25-40, 40$63 \mu \mathrm{m}$; YMC, Japan) and Sephadex LH-20 columns (Pharmacia, Sweden).

Plant material: The whole plant of $C$. japonicus was collected in Taibai mountain of Qinling area, Shaanxi province, P.R.China in 2013, and was identified by Dr. Zhou, Y. F. of Xi'an Botanical Garden, Institute of Botany of Shaanxi. A voucher sample (NO.03054) was deposited at the Plants Herbarium of Institute of Botany of Shaanxi.

[a] Prof. X.-W. Shi, Dr. Q.-Q.Lu, Dr. J.-H. Zhou Xi'an Botanical Garden Institute of Botany of Shaanxi Province Xi'an 710061, Shaanxi, People's Republic of China E-mail: sxw@ms.xab.ac.cn

[b] Prof. G. Pescitelli, Dr. T. Ivšić Dipartimento di Chimica e Chimica Industriale Università di Pisa via Moruzzi 13, I-56124 Pisa, Italy E-mail: gennaro.pescitelli@unipi.it

[c] Dr. T. IVšić

Division of Organic Chemistry and Biochemistry Ruđer Bošković Institute 10000 Zagreb, Croatia

[b] Prof. J.-M. Gao

Shaanxi Key Laboratory of Natural Products \& Chemical Biology, College of Science Northwest A\&F University Yangling 712100, Shaanxi, People's Republic of China E-mail: jinminggao@nwsuaf.edu.cn Published online: ((will be filled in by the editorial staff)) 
Extraction and Isolation: The air-dried roots and aerial parts of $C$. japonicus $(20 \mathrm{~kg}$ ) were extracted three times with $\mathrm{MeOH}(150 \mathrm{~L})$ after 2-hour reflux, and the filtrate was evaporated under reduced pressure. The residue $(1500 \mathrm{~g})$ was diluted with $\mathrm{H}_{2} \mathrm{O}(5$ $\mathrm{L})$ and successively extracted with petroleum ether (PE), EtOAc, and $n-\mathrm{BuOH}(5 \mathrm{~L})$ five times. After evaporation, the PE $(250 \mathrm{~g})$, EtOAc (393 g), and $n$-BuOH (390 g) extracts were obtained. The EtOAc extract was chromatographed over a silica gel column $(P E-E t O A c, 10: 0 \rightarrow 0: 10)$ to yield seven fractions, A-G. Fraction B $(36 \mathrm{~g})$ was subjected to $\mathrm{MCl}$ gel column chromatography $\left(\mathrm{MeOH}-\mathrm{H}_{2} \mathrm{O}, 1: 9 \rightarrow 3: 7 \rightarrow 5: 5 \rightarrow 7: 3 \rightarrow 9: 1\right)$ to yield five fractions $\mathrm{B}_{1}$ $\mathrm{B}_{5}$. The fraction $\mathrm{B}_{2}(6.5 \mathrm{~g})$ eluted with $60 \% \mathrm{MeOH}$ was separated repeatedly by Sephadex LH-20 (MeOH), RP-18 column $(\mathrm{MeOH}-$ $\left.\mathrm{H}_{2} \mathrm{O}, 3: 7 \rightarrow 4: 6 \rightarrow 5: 5 \rightarrow 7: 3 \rightarrow 9: 1\right)$, followed by purification with a silica gel column (PE-EtOAc-CHCl $3,6: 1: 1 \rightarrow 5: 1: 1 \rightarrow 4: 1: 1 \rightarrow 3: 1: 1$ ) to afford compounds 1 (3 mg), 2 (20 mg) and 3 (55 mg).

Multistalide $C(\mathbf{1})$ : colorless oil; $[\alpha]_{D}{ }^{23}=-165.5(c=1.5$ in $\mathrm{MeOH}) ;{ }^{1} \mathrm{H}$ NMR and ${ }^{13} \mathrm{C}$ NMR, see Table 1 ; IR $(\mathrm{KBr}): \mathrm{v}=3361$, 3288, 2922, 2839, 1652, 1386, $1028 \mathrm{~cm}^{-1}$; UV-vis $(\mathrm{MeOH}): \lambda_{\max }$ $(\log \varepsilon)=335$ (0.63), $237 \mathrm{~nm}(3.9) ; \mathrm{ECD}(\mathrm{MeOH}) \lambda_{\max }(\Delta \varepsilon)=342$ $(-5.55), \quad 266(+20.1), 224 \mathrm{~nm}(-13.3)$; ESI-MS $(\mathrm{m} / \mathrm{z}): 617.2$ $[\mathrm{M}+\mathrm{Na}]^{+}$; HR-MS (ESI, $\left.\mathrm{m} / \mathrm{z}\right):[\mathrm{M}+\mathrm{Na}]^{+}$calcd for $\mathrm{C}_{33} \mathrm{H}_{38} \mathrm{O}_{10} \mathrm{Na}$, 617.2465; found 617.2355. All spectra are shown in the Supporting Information.

Shizukaol C (2) and Shizukaol D (3): see Supporting Information.

Brine Shrimp Larvae Lethality Assay: Brine shrimp eggs (Ocean Star International, Inc., USA) were hatched in a large beaker containing artificial seawater $(2.5 \%, \mathrm{pH} 8.0-8.5)$ and were cultured at $28^{\circ} \mathrm{C}$ for $48 \mathrm{~h}$. Compounds 1-3 were dissolved in dimethyl sulfoxide (DMSO) at the concentration of $2 \mathrm{mg} / \mathrm{mL}$, and then transferred in portions of $10,8,6,4,2,1$, and $0.5 \mu \mathrm{L}$ to a 96-well plate, and diluted with artificial seawater (15-20 nuclei larvae) to final volumes of $200 \mu \mathrm{L}$, using DMSO as a blank control and pdophyllotoxin as a positive control. Each test was conducted in triplicate. After $24 \mathrm{~h}$ incubation, the number of dead shrimp in each well was counted under a microscope. The mortality rate was calculated using the formula: $M=[(A-B-N) /(G-$ $\mathrm{N})] \times 100$ where $M=$ percent of the dead larvae after $24 \mathrm{~h} ; \mathrm{A}=$ number of the dead larvae after24 h; $B=$ average number of the dead larvae in the blank control after $24 \mathrm{~h} ; \mathrm{N}=$ number of the dead larvae before starting the test; $G=$ number of selected larvae for test. In addition, the $\mathrm{LC}_{50}$ values were calculated.

Computational Section: MMFF and preliminary DFT calculations were run with Spartan'14 (Wavefunction, Inc., Irvine CA, 2014), with standard parameters and convergence criteria. DFT and TDDFT calculations were run with Gaussian' $09,{ }^{22}$ with default grids and convergence criteria. Conformational searches were run with the Monte Carlo algorithm implemented in Spartan'14 using Merck molecular force field (MMFF). All structures thus obtained were optimized with DFT method using B3LYP functional and 6-31G(d) basis set in vacuo. TDDFT calculations were run using CAM-B3LYP and $\omega$ B97X-D functionals and TZVP basis set, including 40 excited states (down to $159 \mathrm{~nm}$ with CAM-B3LYP). The two functionals led to coincident results (see Supporting Information). ECD spectra were generated using the program SpecDis ${ }^{23}$ by applying a Gaussian band shape with 0.4$0.5 \mathrm{eV}$ exponential half-width, from dipole-length rotational strengths. Transition density plots were built using a locally modified version of the Gaussian 09 development program.

\section{Results and Discussion}

\section{STRUCTURAL IDENTIFICATION OF COMPOUNDS}

Shizukaols C (2) and D (3) (Chart 1) were identified by comparison of their spectroscopic data $(1 \mathrm{H}$ and $13 \mathrm{C}$ NMR, HRESI-MS; see Supporting Information) with reported values. ${ }^{24}$ These two metabolites are reported from this plant for the first time.
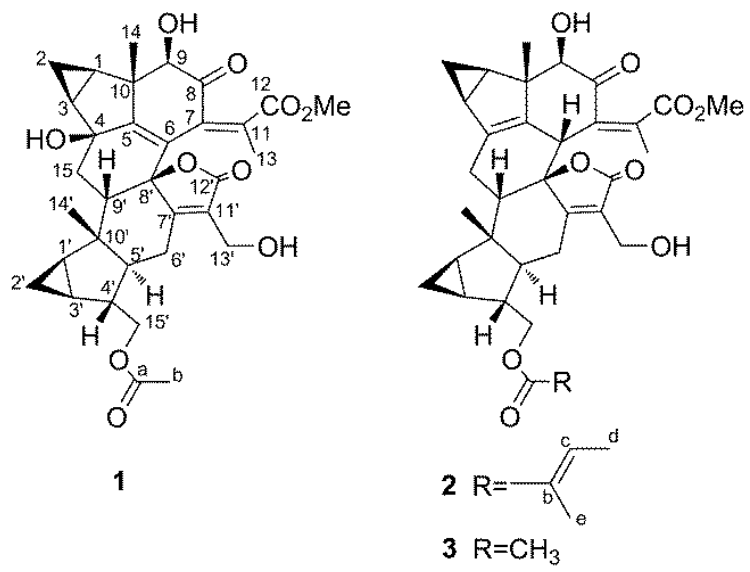

\section{CHART 1 Compounds 1-3.}

TABLE $1{ }^{1} \mathrm{H}$ NMR $(500 \mathrm{~Hz})$ and ${ }^{13} \mathrm{C}$ NMR $(125 \mathrm{MHz})$ data of compound 1 in $\mathrm{CHCl}_{3}-d_{1}: \mathrm{CH}_{3} \mathrm{OH}-d_{4}(3: 1)$.

\begin{tabular}{|c|c|c|c|c|c|}
\hline$\#$ & $\begin{array}{c}\delta_{\mathrm{H}}, \text { mult } \\
(\mathrm{J}, \mathrm{Hz})\end{array}$ & $\bar{\delta}_{\mathrm{C}}$ & $\#$ & $\begin{array}{c}\delta_{H}, \text { mult } \\
(J, \mathrm{~Hz})\end{array}$ & $\bar{\delta}_{\mathrm{C}}$ \\
\hline 1 & $\begin{array}{c}1.99 \mathrm{dd} \\
(12.5,6.3)\end{array}$ & $25.9 d$ & $1^{\prime}$ & $1.26 \mathrm{~m}$ & $26.1 \mathrm{~d}$ \\
\hline $2 \beta$ & $\begin{array}{l}0.95 \mathrm{~d}(4.8) \\
0.93 \mathrm{~d}(7.6)\end{array}$ & $8.6 \mathrm{t}$ & $\begin{array}{l}2^{\prime} \alpha \\
2^{\prime} \beta\end{array}$ & $\begin{array}{c}0.72 \text { dd } \\
(14.3,8.7) \\
0.66 \mathrm{~d}(3.7)\end{array}$ & $16.4 \mathrm{t}$ \\
\hline 3 & $\begin{array}{c}1.85 \mathrm{dd} \\
(12.0,6.4)\end{array}$ & $30.1 \mathrm{~d}$ & $3^{\prime}$ & $1.16 \mathrm{~m}$ & $22.6 \mathrm{~d}$ \\
\hline 4 & & $79.4 \mathrm{~s}$ & $4^{\prime}$ & $1.42 \mathrm{~m}$ & $44.2 \mathrm{~d}$ \\
\hline 5 & & $162.5 \mathrm{~s}$ & $5^{\prime}$ & $1.58 \mathrm{~m}$ & $53.0 \mathrm{~d}$ \\
\hline 6 & & $125.3 \mathrm{~s}$ & $\begin{array}{l}6 ' \alpha \\
6^{\prime} \beta\end{array}$ & $\begin{array}{l}2.59 \mathrm{~m} \\
2.46 \mathrm{~m}\end{array}$ & $25.8 \mathrm{t}$ \\
\hline 7 & & $141.2 \mathrm{~s}$ & $7^{\prime}$ & & $167.5 \mathrm{~s}$ \\
\hline 8 & & $199.6 \mathrm{~s}$ & $8^{\prime}$ & & $87.6 \mathrm{~s}$ \\
\hline 9 & $3.81 \mathrm{~s}$ & $77.6^{a} \mathrm{~d}$ & $9^{\prime}$ & $\begin{array}{c}2.68 \mathrm{dd} \\
(20.3,8.4)\end{array}$ & $54.1 \mathrm{~d}$ \\
\hline 10 & & $50.8 \mathrm{~s}$ & $10^{\prime}$ & & $44.7 \mathrm{~s}$ \\
\hline 11 & & $130.1 \mathrm{~s}$ & $11^{\prime}$ & & $128.4 \mathrm{~s}$ \\
\hline 12 & & $171.2 \mathrm{~s}$ & $12^{\prime}$ & & $172.5 \mathrm{~s}$ \\
\hline 13 & $1.74 \mathrm{~s}$ & $20.9 q$ & $13^{\prime} \alpha$ & $4.36 \mathrm{~d}(13.8)$ & $54.5 \mathrm{t}$ \\
\hline 14 & $1.09 \mathrm{~s}$ & $15.8 q$ & $13^{\prime} \beta$ & $4.31 \mathrm{~d}(13.8)$ & \\
\hline $\begin{array}{l}15 \alpha \\
15 \beta\end{array}$ & $\begin{array}{c}2.75 \mathrm{dd} \\
(13.4,6.9) \\
1.74 \mathrm{~m}\end{array}$ & $41.0 \mathrm{t}$ & $14^{\prime}$ & $0.85 \mathrm{~s}$ & $22.8 \mathrm{q}$ \\
\hline$a$ & & $172.1 \mathrm{~s}$ & $\begin{array}{l}15^{\prime} \alpha \\
15^{\prime} \beta\end{array}$ & $\begin{array}{c}4.00 \mathrm{dd} \\
(11.1,3.9) \\
3.91 \mathrm{dd} \\
(11.1,6.4)\end{array}$ & $66.5 \mathrm{t}$ \\
\hline b & $2.08 \mathrm{~s}$ & $20.7 q$ & & & \\
\hline OMe & $3.79 \mathrm{~s}$ & $52.7 \mathrm{q}$ & & & \\
\hline
\end{tabular}

Compound 1 (Chart 1) was obtained as a colorless oil with an optical rotation of $[\alpha]^{23}-165.5(c=1.5, \mathrm{MeOH})$. The molecular formula $\mathrm{C}_{33} \mathrm{H}_{38} \mathrm{O}_{10}$ was determined by HR-ESI-MS showing the sodiated species at $\mathrm{m} / \mathrm{z} 617.2355 \quad[\mathrm{M}+\mathrm{Na}]^{+}$(calcd. for $\left.\mathrm{C}_{33} \mathrm{H}_{38} \mathrm{O}_{10} \mathrm{Na}, 617.2465\right)$ corresponding to 15 degrees of unsaturation. IR spectra revealed the presence of hydroxyl (3361 $\mathrm{cm}^{-1}$ ) and carbonyl $\left(1652 \mathrm{~cm}^{-1}\right)$ functionalities. The ${ }^{13} \mathrm{C}$ NMR and 
DEPT spectra of 1 (Table 1) showed 33 signals, consisting of four carbonyls $\left(\delta_{c} 199.6,172.5,172.1\right.$ and 171.2), four methyls including an acetoxyl $\left(\delta_{C} 20.7\right)$, one methoxyl $\left(\delta_{C} 52.7\right)$, three persubstituted double bonds, six $\mathrm{sp}^{3}$ methylenes, eight $\mathrm{sp}^{3}$ methines, and four $\mathrm{sp}^{3}$ quaternary carbons. The ${ }^{1} \mathrm{H}$ NMR spectrum (Table 1) showed four methyl protons at $\delta_{H} 0.85$ (s), $1.09(\mathrm{~s}), 1.74(\mathrm{~s})$ and $2.08(\mathrm{~s})$, one methoxyl at $\delta_{\mathrm{H}} 3.79(\mathrm{~s})$, and one $\mathrm{sp}^{3}$ methine proton at $\delta_{\mathrm{H}} 3.81$ (s). The ${ }^{1} \mathrm{H}$ and ${ }^{13} \mathrm{C}$ NMR data of $\mathbf{1}$ were very similar to those of $\mathbf{3}$, suggesting the same skeleton. The major differences between them were that the double bond between $C-4\left(\delta_{C} 142.5\right)$ and $C-5\left(\delta_{C} 131.6\right)$ in 3 shifted to $C-5\left(\delta_{C} 162.5\right)$ and $C-6\left(\delta_{C} 125.3\right)$ in 1 , with an additional hydroxyl appearing at the quaternary carbon $(C-4)\left(\delta_{C}\right.$ 79.4 ) in 1. The HMBC correlations of $\mathrm{H}-14$ and $\mathrm{H}-15$ to $\mathrm{C}-5$, and of $\mathrm{H}-9$ to $\mathrm{C}-5, \mathrm{C}-6$ revealed the presence of the double bond at $\mathrm{C}-5$ and $\mathrm{C}-6$ in 1; the location of the $\mathrm{OH}$ group was supported by HMBC correlations of $\mathrm{H}-2$ to $\mathrm{C}-3$ and $\mathrm{C}-4$, and of $\mathrm{H}-15$ to $\mathrm{C}-4$ (Figure 1). The analysis described above and the previous studies on sesquiterpenoid dimers within this genus suggested that the compound $\mathbf{1}$ is of lindenane type. ${ }^{18,24}$

Analysis of 1D and 2D NMR (HSQC, HMBC, ${ }^{1} \mathrm{H}-{ }^{1} \mathrm{H}$ COSY and NOESY) spectral data allowed for the final structural assignment of 1. The ${ }^{1} \mathrm{H}-{ }^{1} \mathrm{H}$ COSY data (Figure 1) established two sets of typical proton spin systems of two 1,2-disubstituted cyclopropane rings $\left[\mathrm{H}-1: \delta_{\mathrm{H}} 1.99(\mathrm{dd}, 1 \mathrm{H}, J=12.5,6.3 \mathrm{~Hz}, \mathrm{H}-1)\right.$; $\mathrm{H}-2 \alpha$ : $\delta_{\mathrm{H}} 0.95(\mathrm{~d}, 1 \mathrm{H}, \mathrm{J}=4.8 \mathrm{~Hz}), \mathrm{H}-2 \beta: \delta_{\mathrm{H}} 0.93(\mathrm{~d}, 1 \mathrm{H}, J=7.6 \mathrm{~Hz}$ ); $\mathrm{H}-3: \delta_{\mathrm{H}} 1.85(\mathrm{dd}, 1 \mathrm{H}, J=12.0,6.4 \mathrm{~Hz})$ and $\mathrm{H}-1$ ': $\delta_{\mathrm{H}} 1.26(\mathrm{~m}, 1 \mathrm{H})$; H-2' $\alpha$ : $\delta_{H} 0.72(d d, 1 H, J=14.3,8.7 \mathrm{~Hz}), H-2 ' \beta$ : $\delta_{H} 0.66(d, 1 H$, $\left.J=3.7 \mathrm{~Hz}) ; \mathrm{H}-3^{\prime}: \delta_{H} 1.16(\mathrm{~m}, 1 \mathrm{H})\right]$. The connectivity of the two substructures and most of the other functional groups was accomplished by means of HMBC correlations (Figure 2). The NOESY correlation of $\mathrm{H}-3 \alpha / \mathrm{H}_{2}-15$ suggested that $4-\mathrm{OH}$ was $\beta$ oriented. ${ }^{18}$ Additionally, the NOESY correlations between $\mathrm{H}-9, \mathrm{H}-$ 15 and $\mathrm{H}-\mathrm{9}^{\prime}$ are characteristic of the folding of the polycyclic structure of 1 . On the basis of overall NMR evidence, the structure of 1 was determined as shown in Figure 1 and named multistalide $\mathrm{C}$.

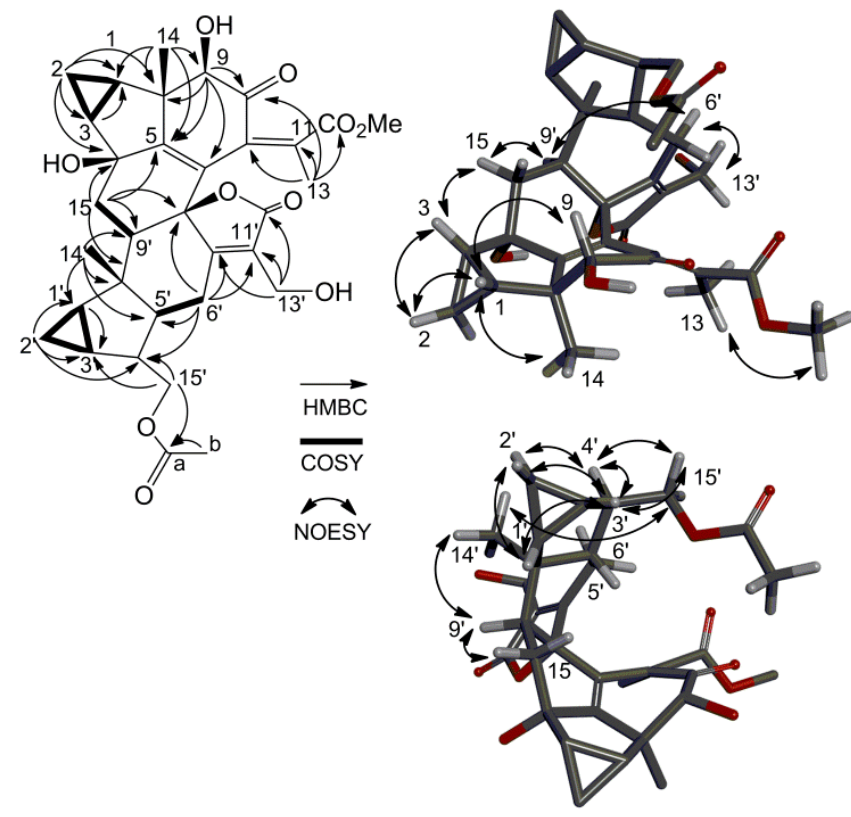

FIGURE 1 Selected HMBC, COSY and NOESY correlations of compound 1 . In the NOESY diagrams (right), the same calculated lowest-energy structure of $\mathbf{1}$ is shown in two different orientations to emphasize the correlations in different molecular portions.
Shizukaol D (3) and multistalide C (1) are biosynthetically related by double bond migration from $\Delta^{4(5)}$ to $\Delta^{5(6)}$ and oxidation at C$4{ }^{25,26}$ Shizukaol $D$ is probably biogenetically generated from shizukaol $A^{7}$ by oxidation at $C-13^{\prime}$ and water addition at $C-4^{\prime} / C$ $15^{\prime}$ double bond. Therefore, the biogenetic pathway to shizukaol D (3) and multistalide C (1) involves the same monomeric precursors producing shizukaol A by Diels-Alder reaction, ${ }^{7,24,27}$ which include shizukanolide $B$ (also known as chloranthalactone A $)^{28}$ as the dienofile.

The absolute configuration (AC) of $\mathbf{1}$ was determined by means of an established protocol based on electronic circular dichroism (ECD). ${ }^{29,30}$ The experimental ECD spectrum of (-)-1 was recorded in methanol (Figure 2) and compared with the spectrum calculated with TDDFT method as Boltzmann average on a set of structures representing low-energy conformers. These latter were obtained by means of a molecular-mechanics conformational search followed by geometry optimizations with DFT method at B3LYP/6-31G(d) level, which afforded 8 structures with relative energies below $3 \mathrm{kcal} / \mathrm{mol}$ and Boltzmann population $>0.2 \%$ at $300 \mathrm{~K}$. In these energy minima (the most stable one is shown in Figure 1, all others are shown in The Supporting Information), the conformation of the relatively rigid polycyclic skeleton is well preserved. The minima differ in the orientation of the flexible chains attached at C-4' and of the hydroxyl groups, and, more importantly, in the orientation of the methyl ester group attached at $\mathrm{C}-11$. This ester moiety is almost perpendicular to the $\mathrm{C} 5-\mathrm{C} 11$ diene system - which itself is substantially twisted - and has two possible conformations with the carbonyl pointed in two opposite directions (see structures in the Supporting Information).

TDDFT calculations were run with CAM-B3LYP and $\omega B$ B7X-D functionals, which currently seem to provide the most accurate prediction of ECD spectra of moderately complex molecules. ${ }^{31,32}$ The Boltzmann-weighted average ECD spectrum calculated with CAM-B3LYP/TZVP for $\left(1 R, 3 S, 4 S, 9 R, 10 S, 1^{\prime} R, 3^{\prime} S, 4^{\prime} R, 5^{\prime} S, 8^{\prime} R\right.$, $9 ' S, 10 ' S)-1$ is shown in Figure 2 and it is in very good agreement with the experimental ECD spectrum measured for (-)-1, therefore the absolute configuration of multistalide $C$ is safely assigned.

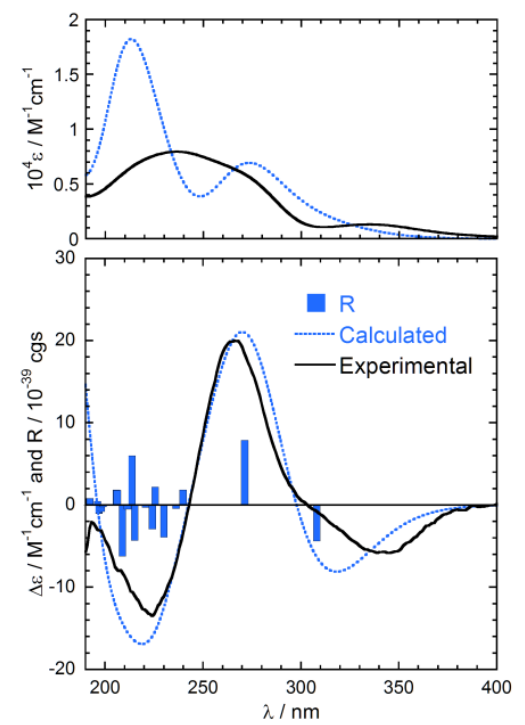

FIGURE 2 Experimental UV (top) and ECD spectra (bottom) of (-)-1 measured in $\mathrm{MeOH}(0.34 \mathrm{mM}, 0.05 \mathrm{~cm}$ cell) compared with the spectra calculated for $\left(1 R, 3 S, 4 S, 9 R, 10 S, 1^{\prime} R, 3^{\prime} S, 4^{\prime} R, 5^{\prime} S, 8^{\prime} R, 9^{\prime} S\right.$, 10'S)-1 at CAM-B3LYP/TZVP level on B3LYP/6-31G(d) geometries, as Boltzmann average at $300 \mathrm{~K}$ for all structures with population $>1 \%$. Gaussian band-shape with $0.4 \mathrm{eV}$ exponential half-width. Vertical 
bars represent rotational strengths calculated for the lowest-energy structure.

\section{ABSOLUTE CONFIGURATION OF CHLORAHOLOLIDE A AND RELATED LINDENANE SESQUITERPENOID DIMERS}

The established absolute configuration of multistalide $C$ is the same for the corresponding chirality centers not only of its biosynthetic precursor chloranthalactone $A,{ }^{28}$ but also of several related sesquiterpenoid dimers sharing the same heptacyclic core. For only a few members of this family, the AC's have been determined by X-ray crystallography ${ }^{11,33}$ or total synthesis. ${ }^{26}$ Most commonly, however, the AC has been assigned on the basis of ECD spectroscopy by applying the exciton chirality method (ECM), ${ }^{25,34-41}$ following a report concerning chlorahololide A (4, diagram in Figure 3 ) where this method was applied for the first time to this class of compounds. ${ }^{18}$ However, we argue here that the ECM not suitable for chlorahololide A and related compounds for several reasons explained below, and reconsider the ECD spectrum and the $A C$ assignment of this compound. Chlorahololide A (4) has the same heptacyclic core as multistalide $C(\mathbf{1})$ and the same main chromophores, namely the unsaturated $\gamma$-lactone and the conjugated $\pi$-electron system spanning carbon atoms $\mathrm{C}-5$ to $\mathrm{C}-8, \mathrm{C}-11$ and $\mathrm{C}-12$, while it differs from 1 in the attachment of the acetate group at C-6', the C-4'/C-15' methylene group, and the absence of C-8 hydroxyl group. Not surprisingly therefore, the ECD spectra of compounds 1 and 4 are very similar (Figure 2 and 3). Applying the same computational protocol described above, we found for $\mathbf{4}$ only two DFT low-energy structures populated at $300 \mathrm{~K}$, differing (as in the case of 1 ) in the orientation of the methyl ester group attached at C-11 (see structures in the Supporting Information). The Boltzmann-weighted average ECD spectrum calculated with CAM-B3LYP/TZVP for $\left(1 R, 3 S, 4 S, 10 S, 1^{\prime} R, 3^{\prime} S, 5^{\prime} S, 6^{\prime} R, 8^{\prime} S, 9^{\prime} S\right.$, 10 'S)-4 is shown in Figure 3 . The agreement with the experimental spectrum confirms the previously determined absolute configuration. ${ }^{18,26}$ This latter was originally based on the application of the non-degenerate exciton chirality method ${ }^{19-21}$ after assigning the two strongest bands in the ECD spectrum, with maxima at $220 \mathrm{~nm}$ and $255 \mathrm{~nm}$, to the $\pi-\pi^{*}$ transitions of the unsaturated $\gamma$-lactone and the twisted $\pi$-electron system, respectively. The two transition moments, drawn in Figure 4a, would define a positive chirality and hence a positive ECD exciton couplet as found experimentally. ${ }^{18}$
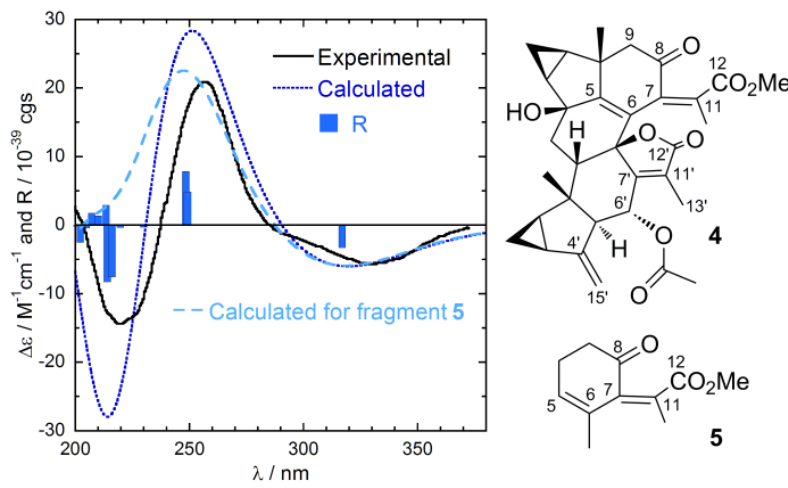

FIGURE 3 Experimental ECD spectrum of (-)-4 in $\mathrm{MeOH}$ (adapted with permission from ref. 18. Copyright 2007 American Chemical Society) compared with the spectrum calculated for $\left(1 R, 3 S, 4 S, 10 S, 1^{\prime} R, 3^{\prime} S, 5^{\prime} S, 6^{\prime} R, 8^{\prime} S, 9^{\prime} S, 10^{\prime} S\right)$-4 at CAM-B3LYP/TZVP level on B3LYP/6-31G(d) geometries, as Boltzmann average at $300 \mathrm{~K}$ for all structures with population $>1 \%$. The dashed line is the spectrum calculated for the fragment 5 in the same conformations found for 4. Gaussian band-shape with $0.5 \mathrm{eV}$ exponential half-width.
Vertical bars represent rotational strengths calculated for the lowestenergy structure.

However, a correct application of the exciton chirality method, including the situation when non-equivalent chromophores are concerned (non-degenerate exciton coupling), necessitates some essential prerequisites: ${ }^{19-21,42}$

(a) The molecular conformation, with special attention to the inter-chromophoric arrangement, must be known with accuracy; ${ }^{29}$

(b) The two electric-dipole allowed transitions, exciton-coupled to each other, should be well separated in energy from other transitions, that is, the two ECD bands must be clearly assigned to the considered chromophores;

(c) The direction of the two electric transition moments must be exactly known;

(d) The two chromophores should be intrinsically achiral, which, in the case of conjugated $\pi$-systems, essentially means planar, to minimize ECD signals not due to the exciton coupling.

A careful analysis of our calculation results on chlorahololide A (4) and of the original paper ${ }^{18}$ clearly reveals that none of the above criteria is really satisfied in the ECM application to this compound. In fact:

(a') The molecular conformation used to establish the chirality between the transition dipoles (Figure $4 a),{ }^{18}$ although possibly generated by molecular modeling and apparently respectful of NOE contacts, has apparently not been ascertained by conformational analysis and geometry optimizations;

(b') As seen in Figure 3, the ECD band between $200-240 \mathrm{~nm}$ is due to the superposition of several different transitions, and none of them can be assigned in a prevalent way to the unsaturated $\gamma$ lactone $\pi-\pi^{*}$ transition;

(c') The $\pi$-electron system involving carbons C-5-C-8, C-11 and $\mathrm{C}-12$ is a quite complicated chromophore, and the direction of the electric transition moment for its $255 \mathrm{~nm}$ band cannot be taken for granted. In the quoted paper, ${ }^{18}$ this is drawn as shown in Figure $4 a$, that is with an arrow going approximately from the middle of the C-5/C- 6 double bond to the middle of ester $\mathrm{C}=\mathrm{O}$ bond, almost neglecting the contribution from the C-8 carbonyl group. However, a transition density plot of such a transition (Figure 4b), calculated on a molecular fragment (5) representing the chromophore in the same conformation found for $\mathbf{4}$, reveals that the transition is delocalized on the whole conjugated moiety, including the C-8 carbonyl group, and the correct direction of the transition moment is rather parallel to the exocyclic $\mathrm{C}-7 / \mathrm{C}-11$ double bond (Figure 4c);

(d') The same $\pi$-electron system is not planar but heavily twisted around both $\mathrm{C}-6 / \mathrm{C}-7$ and $\mathrm{C}-11 / \mathrm{C}-12$ single bonds. The values measured on DFT geometries are $121-124^{\circ}$ for the $\mathrm{C}-5 / \mathrm{C}-11$ dihedral angle (both conformers) and $-111^{\circ}$ and $+76^{\circ}$ for the $\mathrm{C}$ $7 / \mathrm{O}(=\mathrm{C})$ dihedral angle (lowest energy and second energy minimum, respectively). As a consequence, such a chromophore is definitely intrinsically chiral. In fact, both the $n-\pi^{*}$ and $\pi-\pi^{*}$ transitions calculated for fragment $\mathbf{5}$ are associated with strong rotational strengths which account almost entirely for the ECD of compound 4 above $250 \mathrm{~nm}$ (see dotted and dashed lines in Figure 3). 
(a)

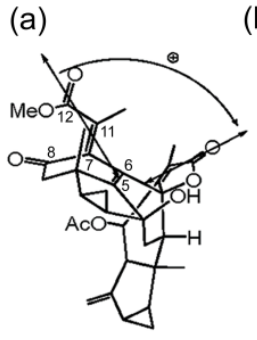

(c)

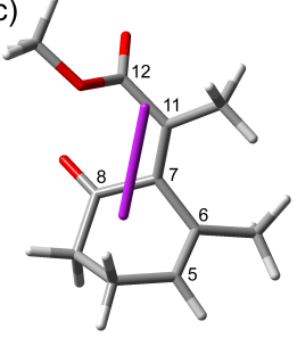

FIGURE 4 (a) Application of the ECM to chlorahololide A (4): the arrows depict the supposed directions of the electric dipole moments (adapted with permission from ref. ${ }^{18}$. Copyright 2007 American Chemical Society). (b,c) Transition density plot for the first $\pi-\pi^{*}$ band calculated at CAM-B3LYP/TZVP level for fragment $\mathbf{5}$ (Figure 3 ) in the same lowest-energy conformation found for $\mathbf{4}$, and direction of the electric transition dipole moment.

In summary, the couplet-like feature appearing in the ECD spectrum of multistalide $C(\mathbf{1})$ and chlorahololide $A(4)$ between $200-290 \mathrm{~nm}$ is not due to the exciton coupling between the two above discussed transitions and cannot be used for a configurational assignment based on the exciton chirality method. The same reasoning applies to all configurational assignments reported in the literature for several related sesquiterpenoid dimers, all of which were based on a similar exciton chirality analysis plagued by the same faults: in all cases, none of the requisites (a)-(d) above is satisfied, and the problems corresponding to points (a')-(d') above are encountered. ${ }^{25,34-41}$ In some cases, moreover, ECD spectra of different lindenane-type compounds were correlated in an empirical manner even if the chromophoric systems were not the same among the various compounds. ${ }^{34-38,40}$ In conclusion, we discourage the use of the exciton chirality method and empirical spectral correlations for this class of compounds, and recommend that full ECD calculations are performed.

\section{BRINE SHRIMP LARVAE TOXICITY ASSAY}

The brine shrimp larvae lethality assay is considered as a useful tool for preliminary assessment of toxicity with compounds and extracts. ${ }^{43,44}$ The three compounds 1-3 were evaluated for brine shrimp larvae (Artemia salina) toxicity, and $\mathbf{2}$ and $\mathbf{3}$ showed significant growth inhibitory activities with median lethal concentration $\left(\mathrm{LC}_{50}\right.$ ) values of 19.0 and $11.7 \mu \mathrm{g} / \mathrm{mL}$, respectively, compared with the positive control podophyllotoxin $(6.0 \mu \mathrm{g} / \mathrm{mL})$, whereas 1 displayed weak activity $\left(\mathrm{LC}_{50} 31.2 \mu \mathrm{g} / \mathrm{mL}\right)$.

\section{Conclusions}

A novel dimeric sesquiterpenoid, multistalide $C(\mathbf{1})$, together with two known dimers, shizukaols C (2) and D (3), were isolated from Chloranthus japonicus Sieb for the first time. Compounds 2 and $\mathbf{3}$ showed remarkable growth inhibitory of brine shrimp larvae. The structure of multistalide C (1) was fully elucidated, including its absolute configuration. A literature survey on absolute configurational assignments of related lindenane-type sesquiterpenoid dimers revealed that in almost all cases the exciton chirality method was applied, starting from the first report on chlorahololide A (4). This latter compound was therefore reconsidered and it was demonstrated that its ECD spectrum is not dominated by the exciton coupling mechanism. Other pitfalls in the reported applications of the exciton chirality method to this class of compounds were also noticed. Also in consideration of the relatively rigid heptacyclic skeleton, the absolute

configuration of chlorahololide-type compounds may be easily assigned by means of TDDFT//DFT calculations.

\section{Acknowledgements}

This work was supported by the National Natural Science Foundation of China (Grant No. 31200257), the Overall Science and Technology Innovation program of Shaanxi Province (Grant No. 2012KTCL02-07), the West Light Foundation of The Chinese Academy of Sciences (Grant No. 2012DF05), the Science and Technology Program of Shaanxi Academy of Sciences (Grant No. 2012k-04) and the Science and Technology Research and Development Projects of Shaanxi Province (Grant No. 2013KJXX74). T. I. gratefully acknowledges FP7-REGPOT-2012-2013-1, grant agreement number 316289 - InnoMol. G.P. thanks Lorenzo Cupellini and Daniele Padula (Univresity of Pisa) for density plots.

\section{Supporting information}

Additional supporting information may be found in the online version of this article at the publisher's website.

\section{REFERENCES AND NOTES}

1. Flora of China Editorial Committee. Flora of China (Zhongguo Zhiwu Zhi). Volume 20. Beijing: Science; 1982. p 85-86.

2. Xu Y-J. Phytochemical and Biological Studies of Chloranthus Medicinal Plants. Chem Biodivers 2013;10:1754-1773.

3. Kawabata J, Fukushi E, Mizutani J. Sesquiterpene dimers from Chloranthus japonicus. Phytochemistry 1995;39:121-125.

4. Kawabata J, Fukushi Y, Tahara S, Mizutani J. Studies on the chemical constituents of Chloranthacee plants. Part III. Structures of Novel Sesquiterpene Alcohols from Chloranthus japonicus (Chloranthaceae). Agric Biol Chem 1984;48:713-717.

5. Yuan T, Zhang C-R, Yang S-P, Yin S, Wu W-B, Dong L, Yue J-M. Sesquiterpenoids and Phenylpropanoids from Chloranthus serratus. $\mathrm{J}$ Nat Prod 2008;71:2021-2025.

6. Wu B, Qu H, Cheng Y. New Sesquiterpenes from Chloranthus japonicus. Helv Chim Acta 2008;91:725-733.

7. Kawabata J, Fukushi Y, Tahara S, Mizutani J. Studies on the chemical constituents of Chloranthacee. Part 7. Shizukaol a, a sesquiterpene dimer from Chloranthus japonicus. Phytochemistry $1990 \cdot 29 \cdot 2332-2334$

8. Wang Q-H, Kuang H-X, Yang B-Y, Xia Y-G, Wang J-S, Kong L-Y. Sesquiterpenes from Chloranthus japonicus. J Nat Prod 2011;74:1620.

9. Kuang H-X, Xia Y-G, Yang B-Y, Wang Q-H, Lü S-W. Lignan constituents from Chloranthus japonicus Sieb. Arch Pharm Res 2009;32:329-334.

10. Yim NH, Hwang El, Yun BS, Park KD, Moon JS, Lee SH, Sung ND, Kim SU. Sesquiterpene Furan Compound CJ-01, a Novel Chitin Synthase 2 Inhibitor from Chloranthus japonicus Sieb. Biol Pharm Bull 2008;31:1041-1044.

11. Fang P-L, Cao Y-L, Yan H, Pan L-L, Liu S-C, Gong N-B, Lü Y, Chen $\mathrm{C}-\mathrm{X}$, Zhong H-M, Guo Y, Liu H-Y. Lindenane Disesquiterpenoids with Anti-HIV-1 Activity from Chloranthus japonicus. J Nat Prod 2011;74:1408-1413.

12. Hu R-K, Yan H, Hao X-J, Liu H-Y, Wu J-R. Shizukaol D isolated from Chloranthus japonicus inhibits AMPK-dependent lipid content in hepatic cells by inducing mitochondrial dysfunction. PloS One 2013;8:e73527. 
13. Tang J-J, Zhang F-Y, Wang D-M, Tian J-M, Dong S, Gao J-M. Semisynthesis and Antifeedant Activity of New Derivatives of a Dihydro- $\beta$-Agarofuran from Parnassia wightiana. Int J Mol Sci 2013; $14: 19484$

14. Liu H-W, Yu X-Z, Padula D, Pescitelli G, Lin Z-W, Wang F, Ding K, Lei M, Gao J-M. Lignans from Schisandra sphenathera Rehd. et Wils. and semisynthetic schisantherin A analogues: Absolute configuration, and their estrogenic and anti-proliferative activity. Eur J Med Chem 2013;59:265-273.

15. Wei S-P, Gao J-M, Wu W-J, Ji Z-Q, Zhang J-W. Simultaneous Identification of Multiple Celangulins from the Root Bark of Celastrus angulatus Using High-performance Liquid Chromatography-Diode Array Detector-Electrospray Ionisation-Tandem Mass Spectrometry. Phytochem Anal 2012;23:23-33.

16. Wang D-M, Zhang C-C, Zhang Q, Shafiq N, Pescitelli G, Li D-W Gao J-M. Wightianines A-E, Dihydro- $\beta$-agarofuran Sesquiterpenes from Parnassia wightiana, and Their Antifungal and Insecticidal Activities. J Agric Food Chem 2014;62:6669-6676.

17. Gao J-M, Wu W-J, Zhang J-W, Konishi Y. The dihydro-bagarofuran sesquiterpenoids. Nat Prod Rep 2007;24:1153-1189.

18. Yang S-P, Gao Z-B, Wang F-D, Liao S-G, Chen H-D, Zhang C-R, Hu G-Y, Yue J-M. Chlorahololides A and B, Two Potent and Selective Blockers of the Potassium Channel Isolated from Chloranthus holostegius. Org Lett 2007;9:903-906.

19. Berova N, Harada N, Nakanishi K. Exciton Coupling. In: Lindon JC, editor. Encyclopedia of Spectroscopy and Spectrometry. Oxford: Elsevier; 1999. p 470-488.

20. Harada N, Nakanishi K. Circular Dichroic Spectroscopy - Exciton Coupling in Organic Stereochemistry. Mill Valley, CA: University Science Books; 1983.

21. Harada N, Nakanishi K, Berova N. Electronic CD Exciton Chirality Method: Principles and Applications. In: Berova N, Polavarapu PL, Nakanishi K, Woody RW, editors. Comprehensive Chiroptical Spectroscopy. Hoboken (NJ): John Wiley \& Sons, Inc.; 2012. p 115-166.

22. Frisch MJ, Trucks GW, Schlegel HB, Scuseria GE, Robb MA, Cheeseman JR, Scalmani G, Barone V, Mennucci B, Petersson GA Nakatsuji H, Caricato M, Li X, Hratchian HP, Izmaylov AF, Bloino J, Zheng G, Sonnemberg JL, Hada M, Ehara M, Toyota K, Fukuda R, Hasegawa J, Ishida M, Nakajima T, Honda Y, Kitao O, Nakai H, Vreven T, Montgomery J, J. A., Peralta JE, Ogliaro F, Bearpark M, Heyd JJ, Brothers E, Kudin KN, Staroverov VN, Kobayashi R,

Normand J, Raghavachari K, Rendell A, Burant JC, lyengar SS,

Tomasi J, Cossi M, Rega N, Millam JM, Klene M, Knox JE, Cross JB, Bakken V, Adamo C, Jaramillo J, Gomperts R, Stratmann RE,

Yazyev O, Austin AJ, Cammi R, Pomelli C, Ochterski JW, Martin RL, Morokuma K, Zakrzewski VG, Voth GA, Salvador P, Dannenberg JJ Dapprich S, Daniels AD, Farkas O, Foresman JB, Ortiz JV,

Cioslowski J, Fox DJ. Gaussian 09, Revision D.01. Wallingford CT: Gaussian, Inc.; 2013.

23. Bruhn T, Schaumlöffel A, Hemberger Y, Bringmann G. SpecDis: Quantifying the Comparison of Calculated and Experimental Electronic Circular Dichroism Spectra. Chirality 2013;25:243-249.

24. Kawabata J, Mizutani J. Dimeric sesquiterpenoid esters from Chloranthus serratus. Phytochemistry 1992;31:1293-1296.

25. Kim S-Y, Kashiwada Y, Kawazoe K, Murakami K, Sun H-D, Li SL, Takaishi Y. Spicachlorantins C-F, hydroperoxy dimeric sesquiterpenes from the roots of Chloranthus spicatus. Tetrahedron Lett 2009;50:6032-6035.

26. Lu Y-S, Peng X-S. A Concise Construction of the Chlorahololide Heptacyclic Core. Org Lett 2011;13:2940-2943.

27. Yang L, Yue G, Yuan C, Du B, Deng H, Liu B. Synthetic Studies toward Lindenane-Type Sesquiterpenoid Dimers. Synlett 2014:25:2471-2474.
28. Kawabata J, Tahara S, Mizutani J, Furusaki A, Hashiba N, Matsumoto T. Shizukanolides, Two Sesquiterpenoids from Chloranthus japonicus (Chloranthacese). Agric Biol Chem 1979:43:885-887.

29. Pescitelli G, Di Bari L, Berova N. Conformational aspects in the studies of organic compounds by electronic circular dichroism. Chem Soc Rev 2011;40:4603-4625.

30. Pescitelli G, Kurtán T, Flörke U, Krohn K. Absolute Structural Elucidation of Natural Products-A Focus on Quantum-Mechanical Calculations of Solid-State CD Spectra. Chirality 2009;21:E181-E201.

31. Gunasekaran P, Perumal S, Menéndez JC, Mancinelli M, Ranieri S, Mazzanti A. Axial Chirality of 4-Arylpyrazolo[3,4-b]pyridines. Conformational Analysis and Absolute Configuration. J Org Chem 2014;79:11039-11050

32. Berardozzi R, Guido CA, Capozzi MAM, Cardellicchio C, Di Bari L, Pescitelli G. Circular Dichroism and TDDFT Investigation of Chiral Fluorinated Aryl Benzyl Sulfoxides. Eur J Org Chem 2015;2015:5554-5562.

33. Xu Y-J, Tang C-P, Ke C-Q, Zhang J-B, Weiss H-C, Gesing E-R, Ye Y. Mono- and Di-sesquiterpenoids from Chloranthus spicatus. $J$ Nat Prod 2007;70:1987-1990.

34. Li C-J, Zhang D-M, Luo Y-M, Yu S-S, Li Y, Lu Y. Bissesquiterpenes and diterpenes from Chloranthus henryi. Phytochemistry 2008;69:2867-2874.

35. Yang S-P, Gao Z-B, Wu Y, Hu G-Y, Yue J-M. Chlorahololides C$\mathrm{F}$ : a new class of potent and selective potassium channel blockers from Chloranthus holostegius. Tetrahedron 2008;64:2027-2034.

36. He X-F, Yin S, Ji Y-C, Su Z-S, Geng M-Y, Yue J-M. Sesquiterpenes and Dimeric Sesquiterpenoids from Sarcandra glabra. J Nat Prod 2010;73:45-50.

37. Zhang S, Yang S-P, Yuan T, Lin B-D, Wu Y, Yue J-M. Multistalides $A$ and $B$, two novel sesquiterpenoid dimers from Chloranthus multistachys. Tetrahedron Lett 2010;51:764-766.

38. Kim S-Y, Kashiwada Y, Kawazoe K, Murakami K, Sun H-D, Li SL, Takaishi Y. Spicachlorantins G-J, New Lindenane Sesquiterpenoid Dimers from the Roots of Chloranthus spicatus. Chem Pharm Bull 2011;59:1281-1284.

39. Zhang M, linuma M, Wang J-S, Oyama M, Ito T, Kong L-Y. Terpenoids from Chloranthus serratus and Their Anti-inflammatory Activities. J Nat Prod 2012;75:694-698.

40. Ni G, Zhang H, Liu H-C, Yang S-P, Geng M-Y, Yue J-M. Cytotoxic sesquiterpenoids from Sarcandra glabra. Tetrahedron 2013:69:564-569.

41. Wang P, Luo J, Zhang Y-M, Kong L-Y. Sesquiterpene dimers esterified with diverse small organic acids from the seeds of Sarcandra glabra. Tetrahedron 2015;71:5362-5370.

42. Berova N, Di Bari L, Pescitelli G. Application of electronic circular dichroism in configurational and conformational analysis of organic compounds. Chem Soc Rev 2007;36:914-931.

43. Li X-J, Zhang Q, Zhang A-L, Gao J-M. Metabolites from Aspergillus fumigatus, an Endophytic Fungus Associated with Melia azedarach, and Their Antifungal, Antifeedant, and Toxic Activities. $J$ Agric Food Chem 2012;60:3424-3431.

44. Qin J-C, Zhang Y-M, Gao J-M, Bai M-S, Yang S-X, Laatsch H, Zhang A-L. Bioactive metabolites produced by Chaetomium globosum, an endophytic fungus isolated from Ginkgo biloba. Bioorg Med Chem 2009;19:1572-1574. 


\section{Graphical Abstract}

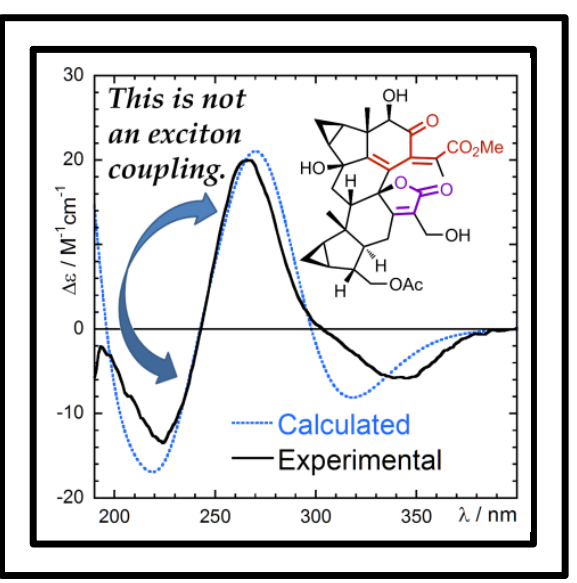

A new sesquiterpenoid dimer, Multistalide C, was isolated from Chloranthus japonicus Sieb and its absolute structure determined. Although displaying a couplet-like feature in the ECD spectrum, the use of exciton chirality method for multistalide $C$ and several related chlorahololide-type sesquiterpenoid dimers reported in the literature is not suitable because of the intrinsic chirality of the composite conjugated chromophore and the complexity of the ECD spectrum. 